\title{
9 STRESS E ENGAGEMENT NA PROFISSÃO DE ENFERMAGEM: ANÁLISE DE DOIS CONTEXTOS INTERNACIONAIS
}

\author{
| Ana Rita Gonçalves'; Ana Galvão²; Susana Escanciano³; Marco Pinheiro ${ }^{4}$; Maria José Gomes ${ }^{5}$ |
}

\begin{abstract}
RESUMO
CONTEXTO: As mudanças constantes nas condições de trabalho, têm conduzido ao aparecimento de riscos psicossociais que conjugados com alterações organizacionais, de gestão de recursos humanos e das cargas horárias, podem levar ao aumento dos níveis de stress e ao comprometimento da saúde física e mental dos trabalhadores.

OBJETIVO(S): Objetivou-se caraterizar duas amostras de enfermeiros (espanhóis e portugueses) e analisar as dimensões do stress e engagement. METODOLOGIA: Estudo comparativo e correlacional. Amostra não probabilística por conveniência, 504 enfermeiros portugueses (65.8\%) e 363 espanhóis (34.2\%) inscritos nas respetivas Ordens.

Utilizou-se um questionário online, composto pela escala de stress, a "Nursing Stress Scale", medindo sete dimensões de stress, e a escala de motivação, a "Utrecht Work Engagement Scale" (UWES), medindo três dimensões de motivação.

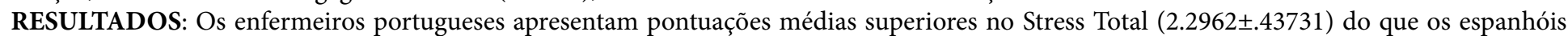

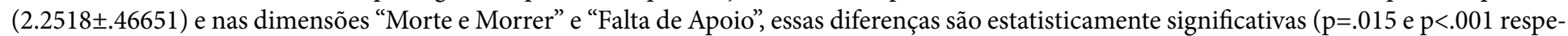
tivamente), sendo que no engagement os espanhóis apresentam resultados consistentemente superiores e estatisticamente significativos ( $<<.001$ ) nas três dimensões. A variável profissional que mais dimensões do stress e do engagement influencia, é o tipo de horário, sendo o horário rotativo mais indutor de stress e menos motivador do que o horário fixo, sendo essas diferenças estatisticamente significativas ( $\mathrm{p} \leq .001)$ para o Stress Total e as três dimensões da UWES.
\end{abstract}

CONCLUSÕES: Existem diferenças a nível do stress e motivação entre os enfermeiros portugueses e espanhóis.

\section{PALAVRAS-CHAVE: Saúde mental; Cuidados de enfermagem; Estresse psicológico; Motivação}

\section{RESUMEN}

\section{“Estrés y engagement en la profesión de enfermería: Análisis de dos contextos internacionales"}

CONTEXTO: Los cambios constantes en las condiciones de trabajo, han conducido a la aparición de riesgos psicosociales que, conjugados con alteraciones organizacionales, de gestión de recursos humanos y de las cargas horarias, pueden conducir al aumento de los niveles de estrés y al comprometimiento de la salud física y mental trabajadores.

OBJETIVO(S): El objetivo fue caracterizar dos muestras de enfermeras (España y Portugal) y el análisis de las dimensiones de estrés y de compromiso. METODOLOGÍA: Estudio comparativo y correlacional. La muestra no probabilística de conveniencia, portugués 504 enfermeras $(65,8 \%)$ y 363 españoles $(34,2 \%)$ inscritos en los respectivos Órdenes. Se utilizó un cuestionario online, compuesto por la escala de estrés, la "Nursing Stress Scale", midiendo siete dimensiones de estrés, y la escala de motivación, la "Utrecht Work Engagement Scale" (UWES), midiendo tres dimensiones de motivación.

RESULTADOS: Las enfermeras portuguesas tienen mayores puntuaciones medias en estrés total $(2,2962 \pm 0,43731)$ que la española $(2,2518 \pm 0,46651)$ y dimensiones "muerte y el morir" y "falta de apoyo", estas diferencias son estadísticamente significativas ( $\mathrm{p}=.015$ e $\mathrm{p}<.001$ respetivamente) y en el compromiso los españoles presentan resultados consistentemente superiores y estadísticamente significativos ( $\mathrm{p}<.001$ ) en las tres dimensiones. La variable profesional que más dimensiones del estrés y del compromiso influye, es el tipo de horario, siendo el horario rotativo más inductor de estrés y menos motivador que el horario fijo, siendo esas diferencias estadísticamente significativas $(\mathrm{p} \leq .001)$ para el Estrés Total y las tres dimensiones de la UWES. CONCLUSIONES: Existen diferencias en el estrés y la motivación entre los enfermeros de Portugal y España.

\section{DESCRIPTORES: Salud mental; Atención de enfermería; Estrés} psicológico; Motivación

\section{ABSTRACT}

"Stress and engagement in the nursing profession: Analysis of two international contexts"

BACKGROUND: Constant changes in working conditions have led to the emergence of psychosocial risks that, combined with organizational changes, human resources management and working hours, can lead to an increase in stress levels and compromise the physical and mental health of workers. AIM: To characterize two samples of nurses (Spanish and Portuguese) and analyse the dimensions of stress and engagement.

METHODS: Comparative and correlational study. Non-probabilistic sample for convenience, 504 Portuguese nurses (65.8\%) and 363 Spaniards (34.2\%) enrolled in the respective Nurses Associations. An online questionnaire was used, consisting of the stress scale, the Nursing Stress Scale, measuring seven dimensions of stress, and the Utrecht Work Engagement Scale (UWES), measuring three dimensions of motivation.

RESULTS: Portuguese nurses have higher mean scores in Total Stress $(2.2962$ $\pm .43731)$ than the Spanish $(2.2518 \pm .46651)$ and in the "Death and Dying" and "Lack of Support" dimensions, those differences are statistically significant ( $\mathrm{p}=.015$ and $\mathrm{p}<.001$ respectively), and in engagement, the Spaniards presented consistently higher and statistically significant scores $(\mathrm{p}<.001)$ in the three dimensions. The professional variable that more dimensions of stress and engagement influences, is the type of schedule, with rotating hours inducing more stress and less motivating than fixed hours, these differences being statistically significant $(\mathrm{p} \leq .001)$ for Total Stress and the three dimensions of UWES.

CONCLUSIONS: There are differences in stress and motivation among Portuguese and Spanish nurses.

\section{KEYWORDS: Mental health; Nursing care; Psychological stress; Motivation}

Submetido em 30-12-2017

Aceite em 30-03-2018

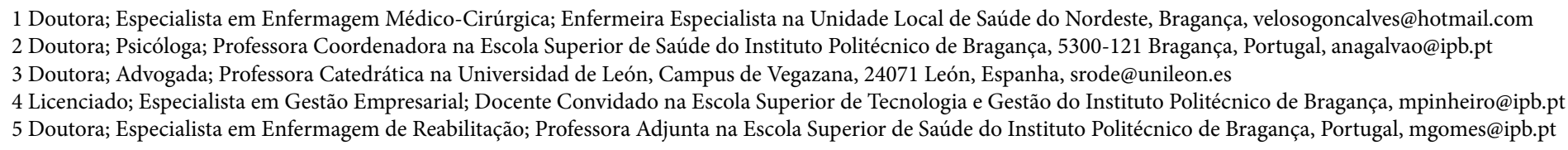

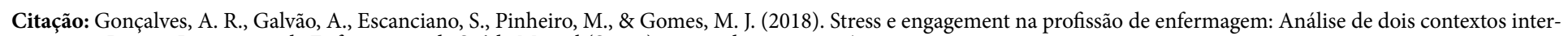
nacionais. Revista Portuguesa de Enfermagem de Saúde Mental (Spe. 6), 59-64. doi: 10.19131/rpesm.0214 


\section{INTRODUÇÃO}

As mudanças constantes nas condições de trabalho, têm conduzido inevitavelmente ao aparecimento de riscos psicossociais (Agência Europeia para a Segurança e Saúde no Trabalho, 2015b). Estes riscos, conjugados com alterações organizacionais, de gestão de recursos humanos e das cargas horárias, podem levar ao aumento dos níveis de stress e, consequentemente, a um comprometimento da saúde física e mental dos trabalhadores.

O estudo das condições organizacionais e psicossociais relacionadas com a saúde dos trabalhadores, foi adquirindo grande importância nos últimos anos, chegando-se à conclusão, que o stress é o segundo problema relacionado com o trabalho mais frequente na Europa (depois das perturbações músculo-esqueléticas), já que $51 \%$ dos trabalhadores referem ser comum o stress laboral na sua organização e 4 em cada 10, referem que o problema não é gerido da melhor forma (Agência Europeia para a Segurança e Saúde no Trabalho, 2015a).

Tanto na União Europeia como nos Estados Unidos, o stress laboral é considerado na atualidade como um dos principais problemas de saúde laboral, com grandes custos económicos e sociais. No que respeita à componente financeira do problema, a Agência Europeia para a Segurança e Saúde no Trabalho, estima que na Europa os custos totais com perturbações de saúde mental (relacionadas ou não com o trabalho) ascendam a 240 mil milhões de Euros por ano, sendo 136 mil milhões de Euros imputáveis à perda de produtividade, incluindo o absentismo por baixa médica.

O engagement no trabalho é definido como: "um estado positivo da mente, realizador e relacionado ao trabalho que é caracterizado pelo vigor, dedicação e absorção" (Schaufeli, Salanova, González-Romá, \& Bakker, 2002, p. 74). Este conceito pode ser considerado como indicativo de uma vida saudável, em relação ao trabalho. Os trabalhadores que obtêm resultados elevados nas escalas que avaliam o engagement evidenciam uma energia muito positiva e estão perfeitamente integrados e motivados com a profissão que desempenham, sentindo-se competentes para resolverem problemas que podem surgir no âmbito do seu trabalho (Rodrigues e Barroso, 2008). Schaufeli e Salanova (2007) caraterizam os trabalhadores engaged como portadores de um sentido de energia e eficácia associadas às suas atividades no trabalho, percecionando-se capazes de lidar com as exigências do trabalho.
São considerados agentes ativos que tomam a iniciativa no trabalho, tentam observar novas mudanças no trabalho, e quando não sentem a mudança preferem mudar de trabalho, adaptando-se rapidamente a novas situações; são indivíduos cujo envolvimento no trabalho se traduz por um elevado nível de qualidade, o qual é normalmente provocado pelo feedback positivo dos supervisores e da organização através de promoções, salários atrativos, e satisfação profissional, e também pelos clientes; são indivíduos que utilizam estratégias de coping eficazes e que trabalham arduamente pois consideram o trabalho agradável, valorizam os aspetos interpessoais e intrínsecos do trabalho e os recursos do mesmo (Salanova \& Schaufeli, 2008).

\section{METODOLOGIA}

Procedeu-se a um estudo comparativo e quantitativo de carater descritivo e correlacional, num plano transversal. $O$ instrumento de recolha de dados foi disponibilizado online a todos os enfermeiros inscritos nas respetivas Ordens Profissionais (portuguesa e espanhola), obtendo-se uma amostra não probabilística por conveniência de 504 enfermeiros portugueses (65.8\%) e 363 enfermeiros espanhóis (34.2\%).

Como instrumento de recolha de dados utilizou-se um questionário, composto por três partes:

- A primeira parte por um questionário sociodemográfico e profissional;

- A segunda parte pela Nursing Stress Scale de GrayToft e Anderson (1981), traduzida e adaptada para a população portuguesa (Santos \& Teixeira, 2008) - Escala de Stresse Profissional dos Enfermeiros (ESPE) e a versão castelhana, traduzida e adaptada para a população espanhola (López Fernández, 2002) - Escala de Estrés de Enfermería, que mede sete dimensões de stress, sendo elas: A morte e o morrer; Conflitos com os médicos; Preparação inadequada para lidar com as necessidades emocionais dos doentes e dos seus familiares; Falta de apoio dos colegas; Conflitos com outros enfermeiros e com os chefes; Carga de trabalho; e Incerteza quanto aos tratamentos.

- A terceira parte pela Utrecht Work Engagement Scale, versão traduzida para a população portuguesa (Schaufeli, Martinez, Pinto, Salanova, \& Bakker, 2002) - Escala de Engagement no Trabalho de Utrecht (UWES) e a Encuesta de Bienestar y Trabajo, versão espanhola da escala original (Schaufeli, Bakker, \& Salanova, 2006), medindo três dimensões: Vigor; Dedicação; e Absorção. 
A caraterização socioprofissional é apresentada na Tabela 1, verificando-se uma amostra composta maioritariamente por respondentes do sexo feminino (83.6\%), com horário rotativo (55.0\%) e uma carga horária de 40 horas (61.4\%).

Tabela 1 - Caraterização Socioprofissional da Amostra

\begin{tabular}{|c|c|c|c|c|c|c|c|}
\hline & & \multicolumn{2}{|c|}{ Portugal } & \multicolumn{2}{|c|}{ Espanha } & \multicolumn{2}{|c|}{ Total } \\
\hline & & $\mathrm{n}$ & $\%$ & $\mathrm{n}$ & $\%$ & $\mathrm{n}$ & $\%$ \\
\hline \multirow[t]{3}{*}{ Género } & $\begin{array}{l}\text { Femi- } \\
\text { nino }\end{array}$ & 396 & $78.6 \%$ & 329 & $90.6 \%$ & 725 & $83.6 \%$ \\
\hline & $\begin{array}{l}\text { Mascu- } \\
\text { lino }\end{array}$ & 108 & $21.4 \%$ & 34 & $9.4 \%$ & 142 & $16.4 \%$ \\
\hline & Total & 504 & $100.0 \%$ & 363 & $100.0 \%$ & 867 & $100.0 \%$ \\
\hline \multirow[t]{5}{*}{$\begin{array}{l}\text { Tipo } \\
\text { horário }\end{array}$} & $\begin{array}{l}\text { Horário } \\
\text { rotativo }\end{array}$ & 290 & $57.5 \%$ & 187 & $51.5 \%$ & 477 & $55.0 \%$ \\
\hline & Fixo & 144 & $28.6 \%$ & 133 & $36.6 \%$ & 277 & $31.9 \%$ \\
\hline & Misto & 64 & $12.7 \%$ & 41 & $11.3 \%$ & 105 & $12.1 \%$ \\
\hline & Outro & 6 & $1.2 \%$ & 2 & $0.6 \%$ & 8 & $0.9 \%$ \\
\hline & Total & 504 & $100.0 \%$ & 363 & $100.0 \%$ & 867 & $100.0 \%$ \\
\hline \multirow{3}{*}{$\begin{array}{l}\text { Carga } \\
\text { horária }\end{array}$} & 35 horas & 86 & $17.1 \%$ & 91 & $25.1 \%$ & 177 & $20.4 \%$ \\
\hline & 40 horas & 389 & $77.2 \%$ & 143 & $39.4 \%$ & 532 & $61.4 \%$ \\
\hline & outro & 29 & $5.8 \%$ & 129 & $35.5 \%$ & 158 & $18.2 \%$ \\
\hline
\end{tabular}

\section{RESULTADOS}

Os resultados, entre os enfermeiros dos dois países, nas sete dimensões de stress medidas, são bastante similares, sendo que no stress total os enfermeiros portugueses têm uma média superior $(2.30 \pm .437)$ do que os espanhóis $(2.25 \pm .467)$, conforme pode ser observado na Tabela 2.

Tabela 2 - Pontuações Médias do Stress Total e Três Dimensões do Engagement

\begin{tabular}{|l|c|c|c|c|c|c|}
\hline \multirow{2}{*}{} & \multicolumn{2}{|c|}{ Portugal } & \multicolumn{2}{c|}{ Espanha } & \multicolumn{2}{c|}{ Total } \\
\cline { 2 - 7 } & $\mathrm{M}$ & $\mathrm{DP}$ & $\mathrm{M}$ & $\mathrm{DP}$ & $\mathrm{M}$ & $\mathrm{DP}$ \\
\hline ESPE Total & 2.30 & .437 & 2.25 & .467 & 2.28 & .45 \\
\hline UWES_Vigor & 3.97 & 1.23 & 4.34 & 1.33 & 4.13 & 1.29 \\
\hline UWES_Dedic & 4.20 & 1.40 & 4.51 & 1.51 & 4.33 & 1.45 \\
\hline UWES_Absor & 3.90 & 1.27 & 4.34 & 1.29 & 4.08 & 1.30 \\
\hline
\end{tabular}

Contudo, conforme apresentado na Tabela 2, em termos de engagement, os enfermeiros espanhóis apresentam resultados consistentemente superiores nas três dimensões.

Na Tabela 3 apresentam-se as pontuações médias dos enfermeiros portugueses e espanhóis para as 4 dimensões sob análise (Stress total; Vigor; Dedicação; e Absorção), bem como os valores de teste de significância obtidos através dos testes de Mann-Whitney efetuados, concluindo-se que as diferenças entre as pontuações medidas para as 3 dimensões do engagement são estatisticamente significativas $(\mathrm{p}<.001)$, não se verificando tal para as diferenças medidas nos níveis de stress.

Tabela 3 - Relações Entre o Stress e o Engagement e o País

\begin{tabular}{|l|l|l|l|}
\hline Dimensão & País & $\mathrm{M}$ & $\mathrm{p}$ \\
\hline \multirow{2}{*}{ ESPE Total } & Portugal & 2.30 & \multirow{2}{*}{.166} \\
\cline { 2 - 3 } & Espanha & 2.25 & \\
\hline \multirow{2}{*}{ UWES-Vigor } & Portugal & 3.97 & \multirow{2}{*}{.000} \\
\cline { 2 - 3 } & Espanha & 4.34 & \\
\hline \multirow{2}{*}{ UWES-Dedicação } & Portugal & 4.20 & \multirow{2}{*}{.000} \\
\cline { 2 - 3 } & Espanha & 4.51 & \\
\hline \multirow{2}{*}{ UWES-Absorção } & Portugal & 3.90 & \multirow{2}{*}{.000} \\
\cline { 2 - 3 } & Espanha & 4.34 & \\
\hline
\end{tabular}

Contudo, conforme poderá ser observado na Tabela 4, verificou-se que em termos de stress, em duas das sete dimensões, "Morte e Morrer" e "Falta de Apoio", os valores superiores verificados nos enfermeiros portugueses são estatisticamente significativos $(\mathrm{p}=.015 \mathrm{e}$ $\mathrm{p}<.001$ respetivamente)

Tabela 4 - Relações entre Stress Provocado pelo Medo da Morte e Morrer e Falta de Apoio e o País

\begin{tabular}{|l|l|l|l|}
\hline Dimensão & País & $\mathrm{M}$ & $\mathrm{p}$ \\
\hline \multirow{2}{*}{ ESPE Morte e Morrer } & Portugal & 2.37 & \multirow{2}{*}{.015} \\
\cline { 2 - 3 } & Espanha & 2.28 & \\
\hline \multirow{2}{*}{ ESPE Falta de Apoio } & Portugal & 2.23 & \multirow{2}{*}{.000} \\
\cline { 2 - 3 } & Espanha & 2.03 & \\
\hline
\end{tabular}

Verifica-se igualmente que a variável profissional que apresenta relação estatisticamente significativa com um maior número de dimensões do stress e do engagement é o tipo de horário, em que o horário rotativo é mais indutor de stress e menos motivador do que um horário fixo, sendo que essas diferenças são estatisticamente significativas $(\mathrm{p} \leq .001)$ nas quatro dimensões medidas, conforme apresentado na Tabela 5.

Tabela 5 - Relações entre as 4 Dimensões Estudadas e o Tipo de Horário

\begin{tabular}{|l|l|l|l|}
\hline Tipo horário & País & $\mathrm{M}$ & $\mathrm{p}$ \\
\hline \multirow{3}{*}{ ESPE Total } & Fixo & 2.17 & \multirow{2}{*}{.000} \\
\cline { 2 - 3 } & Rotativo & 2.35 & \\
\hline \multirow{2}{*}{ UWES_Vigor } & Fixo & 4.34 & \multirow{2}{*}{.000} \\
\cline { 2 - 3 } & Rotativo & 3.98 & \\
\hline UWES_Dedic & Fixo & 4.53 & \multirow{2}{*}{.001} \\
\cline { 2 - 3 } & Rotativo & 4.20 & \\
\hline \multirow{2}{*}{ UWES_Absor } & Fixo & 4.34 & \multirow{2}{*}{.000} \\
\cline { 2 - 3 } & Rotativo & 3.90 & \\
\hline
\end{tabular}


Observa-se, igualmente, uma relação estatisticamente significativa entre as 4 dimensões estudadas, conforme apresentado na Tabela 6.

Tabela 6 - Correlações de Spearman (rho) entre as 4 Dimensões Estudadas

\begin{tabular}{|l|l|l|l|l|}
\hline & $\begin{array}{l}\text { ESPE } \\
\text { Total }\end{array}$ & $\begin{array}{l}\text { UWES_ } \\
\text { Vigor }\end{array}$ & $\begin{array}{l}\text { UWES_ } \\
\text { Dedic }\end{array}$ & $\begin{array}{l}\text { UWES_ } \\
\text { Absor }\end{array}$ \\
\hline ESPE Total & 1.000 & $-.190^{\star *}$ & $-.122^{\star *}$ & $-.089^{\star *}$ \\
\hline UWES_Vigor & & 1.000 & $.834^{\star *}$ & $.847^{\star *}$ \\
\hline UWES_Dedic & & & 1.000 & $.791^{\star *}$ \\
\hline UWES_Absor & & & & 1.000 \\
\hline
\end{tabular}

**. A correlação é significativa no nível 0,01 (bilateral).

De acordo com os dados apresentados na Tabela 6, existe uma relação significativa entre o stress e as 3 dimensões do engagement.

\section{DISCUSSÃO}

Vários estudos concluem haver uma relação entre a profissão de enfermagem e, entre outros, o stress, sendo que concluem igualmente que os níveis de stress em enfermeiros estão relacionados com nomeadamente o tipo de horário de trabalho (Bakker, Schaufeli, Leiter, \& Taris, 2008; Gerber, Hartmann, Brand, HolsboerTrachsler, \& Pühse, 2010).

Outros estudos concluíram que o apoio, em especial das chefias, potencia o engagement e a redução de stress (Cardoso, 2009; Reeve, Shumaker, Yearwood, Crowell, \& Riley, 2013), o que vai ao encontro dos resultados do nosso estudo em que a diferença em níveis de stress provocados pela falta de apoio eram superiores nos enfermeiros portugueses, sendo que estes apresentam níveis de engagement mais baixos.

Vários fatores contribuem para o stress nos enfermeiros, contudo, estudos apontam para uma maior influencia das condições de trabalho do que das vivências da profissão como, por exemplo, o contato com a morte, ou outros fatores que eventualmente podem à primeira vista ser considerados mais stressantes (Galdikiene, Asikainen, Balciunas, \& Suominen, 2016).

Estudos concluem que os fatores indutores de stress mencionados com mais frequência pelos enfermeiros são a falta de recursos, a pressão do tempo e a sobrecarga de trabalho (Rainho, Pimenta, Antunes, \& Monteiro, 2015) e que o conceito de engagement parece funcionar como um fator protetor de stress.
Verifica-se, também, no presente estudo uma relação inversamente correlacional entre o stress e o engagement, este último nas suas três dimensões sob análise, corroborando, assim, os resultados de outros estudos realizados a este nível (Bakker et al., 2008; García-Sierra, Fernández-Castro, \& Martínez-Zaragoza, 2016).

\section{CONCLUSÃO}

Do estudo realizado, verifica-se que o horário é a variável profissional que mais relações estatisticamente significativas apresenta com as várias dimensões estudadas, podendo ser um dos fatores que explica a diferença encontrada entre os enfermeiros dos dois países, já que no caso português somente $28.6 \%$ dos enfermeiros tem um horário fixo, comparando com os $36.6 \%$ dos enfermeiros espanhóis com esse tipo de horário.

Está em análise a proposta de incluir a depressão e o burnout entre as doenças profissionais. A Ordem dos Psicólogos propõe vigilância psicossocial periódica nos locais de trabalho, à semelhança da vigilância regular das doenças físicas, com o objetivo de garantir o bem estar das pessoas e das organizações (Mateus \& Arreigoso, 2017).

A saúde mental de qualquer trabalhador e consequentemente dos enfermeiros, tem um impacto direto no desempenho das suas funções e deverá ser alvo de estudos aprofundados que possam ajudar a minimizar o stress numa profissão que dadas as suas caraterísticas já é por si indutora de stress. Este estudo pretendeu contribuir para o tema da saúde mental, através do conhecimento dos níveis de engagement e stress em enfermeiros de dois contextos internacionais.

\section{IMPLICAÇÕES PARA A PRÁTICA CLÍNICA}

As organizações na atualidade têm demonstrado uma preocupação crescente em proporcionar condições de trabalho no sentido de melhorar a performance dos seus colaboradores e consequentemente a qualidade dos serviços prestados.

O presente estudo, que compara a prática de enfermagem em duas realidades distintas, contribui para melhor entender possíveis causas do aumento de stress e diminuição de engagement no local de trabalho por parte dos enfermeiros. 
Pretende-se, através deste estudo, promover a consciencialização junto dos gestores das unidades de saúde, para uma melhor adequação das condições de trabalho, nomeadamente no que concerne o tipo de horário e a carga horária, por forma a melhorar o desempenho das equipas de enfermagem e consequentemente a qualidade de serviço prestado aos utentes, sem que tal tenha implicações orçamentais, mas somente organizacionais.

Vários são os estudos nos últimos anos que apresentam vários modelos e técnicas para melhor gerir $\mathrm{o}$ stress (Adriaenssens, De Gucht, \& Maes, 2015; Nowrouzi et al., 2015), sendo nossa opinião que tanto as técnicas de gestão de stress bem como suporte profissional através do coaching psicológico, podem trazer benefícios tanto para a qualidade de vida e bem-estar dos enfermeiros, para a melhoria da produtividade das organizações onde prestam serviço e, em última instância, a qualidade dos cuidados de saúde prestados aos utentes. Assim, o Coaching Psicológico visa aumentar o nível de satisfação e de bem-estar, o desempenho na vida pessoal e profissional e reduzir o stress no trabalho, conduzindo a melhores resultados e ao sucesso.

\section{REFERÊNCIAS BIBLIOGRÁFICAS}

Adriaenssens, J., De Gucht, V., \& Maes, S. (2015). Causes and consequences of occupational stress in emergency nurses, a longitudinal study. Journal of Nursing Management, 23 (3), 346-358. Doi: 10.1111/ jonm. 12138 .

Agência Europeia para a Segurança e Saúde no Trabalho. (2015a). Annual Report 2014. Disponível em: https://osha.europa.eu/pt/tools-and-publications/ publications/annual-report-2015-summary

Agência Europeia para a Segurança e Saúde no Trabalho. (2015b). Segundo Inquérito Europeu às Empresas Sobre Riscos Novos e Emergentes - ESENER-2. Disponível em: https://osha.europa.eu/pt/surveys-andstatistics-osh/esener

Bakker, A. B., Schaufeli, W., Leiter, M. P., \& Taris, T. W. (2008). Work engagement: An emerging concept in occupational health psychology. Work \& Stress, 22 (3), 187-200. Disponível em: https://doi. org/10.1080/02678370802393649.
Cardoso, D. (2009). Engagement nos enfermeiros: qual o papel do líder transformacional? (Dissertação de Mestrado, Universidade de Lisboa). Disponível em: http:// repositorio.ul.pt/bitstream/10451/876/1/18756_ulsd_ dep.17740_Dissertacao_Mestrado.pdf

Galdikiene, N., Asikainen, P., Balciunas, S., \& Suominen, T. (2016). Experienced stress among nursing teams in primary health care. Clinical Nursing Studies, 4 (1), 81-90. Disponível em: https://doi.org/10.5430/cns. v4n1p81

García-Sierra, R., Fernández-Castro, J., \& MartínezZaragoza, F. (2016). Work engagement in nursing: An integrative review of the literature. Journal of Nursing Management, 24 (2), 110-111. Doi: 10.1111/ jonm.12312.

Gerber, M., Hartmann, T., Brand, S., HolsboerTrachsler, E., \& Pühse, U. (2010). The relationship between shift work, perceived stress, sleep and health in Swiss police officers. Journal of Criminal Justice, 38 (6), 1167-1175. Disponível em: https://doi.org/10.1016/j. jcrimjus.2010.09.005

Gray-Toft, P., \& Anderson, J. G. (1981). The Nursing Stress Scale: Development of an instrument. Journal of Behavioral Assessment, 3 (1), 11-23. Disponível em: https://link.springer.com/article/10.1007/BF01321348

López Fernández, J. Á. (2002). Validación de la «Escala de Estrés de Enfermería» (NSS), de Gray-Toft y Anderson (Masters Thesis, Universidad de la Laguna). Disponível em: https://www.cop.es/colegiados/T-00921/ tesina.pdf

Mateus, C., \& Arreigoso, V. L. (2017, Dezembro 24). Empresas devem avaliar saúde mental. Expresso - Suplemento Economia.

Nowrouzi, B., Lightfoot, N., Larivière, M., Carter, L., Rukholm, E., Schinke, R., \& Belanger-Gardner, D. (2015). Occupational Stress Management and Burnout Interventions in Nursing and Their Implications for Healthy Work Environments. Workplace Health \& Safety, 63 (7), 308-315. Disponível em: https://doi. org/10.1177/2165079915576931 
